\title{
Acceleration and cyclotron radiation induced by gravitational waves
}

\author{
D. Papadopoulos ${ }^{\star}$ \\ Department of Physics, Aristoteleion University of Thessaloniki, 54006 Thessaloniki, Greece
}

Received 16 July 2002 / Accepted 5 September 2002

\begin{abstract}
The equations that determine the response of a charged particle moving in a magnetic field to an incident gravitational wave (GW) are derived in the linearized approximation to general relativity. We briefly discuss several astrophysical applications of the derived formulae, taking into account the resonance between the wave and the particle's motion that occurs at $\omega_{\mathrm{g}}=2 \Omega$, whenever the GW is parallel to the constant magnetic field. In the case where the GW is perpendicular to the constant magnetic field, magnetic resonances appear at $\omega_{\mathrm{g}}=\Omega$ and $\omega_{\mathrm{g}}=2 \Omega$. Such a resonant mechanism may be useful in building models of GW-driven cyclotron emitters.
\end{abstract}

Key words. relativity - gravitational waves

\section{Introduction}

Papadopoulos \& Esposito (1981) discussed the perturbations of the Larmor orbits in the presence of a gravitational wave (GW) and estimated the resulting magnetic bremsstrahlung. They have shown that it is possible to identify the presence of GWs in macroscopic systems by detecting the shifts in the spectrum of the electromagnetic radiation (cyclotron) given off by charged particles as they interact with a GW.

Recently, the motion of a relativistic charged particle in a constant magnetic field perturbed by a GW incident along the direction of the magnetic field has been examined (van Holten et al. 1999 and references therein). In the same work, a generalized energy conservation law to compute the variations of the kinetic energy of the particle during the passage of the GW has been derived and explicit computations of the orbit of the charged particle due to the GW have been obtained.

In this paper, we discuss the interaction of GW with a charged gyrating particle in the presence of a constant magnetic field across the $z$-axis in the framework of linearized theory of gravity.

When the GW propagates parallel to the magnetic field with a frequency $\omega_{\mathrm{g}}$, the coupling of a gyrating particle with the GW becomes very strong at the resonance that occurs between the GW and the Larmor orbits. The resonance is at twice the Larmor frequency $\left(\Omega=\frac{e H}{m c}\right)$ e.g. $\omega_{\mathrm{g}}=2 \Omega$ (Macedo \& Nelson 1990).

^ e-mail: papadop@astro.auth.gr
In the case that the GW propagates perpendicular to the magnetic field, the interaction again becomes extremely efficient at the resonances, $\omega=\Omega$ and $\omega=2 \Omega$.

In both cases we verify that close to gyro resonances, the obtained spectrum of the produced cyclotron radiation becomes comparable to the spectrum of the initially gyrating particle, especially in the vicinity of a source producing the GW.

Our results suggest that a) the linear theory breaks down at the resonances and the interaction of the GW with gyrating particles becomes very efficient and, b) the linear theory supports the discrepancy on the estimations for the cyclotron damping radiation recently discussed by M. Servin (Servin et al. 2001) and Kleidis (Kleidis et al. 1996; Kleidis et al. 1995), since in Kleidis work, the problem is examined in the non-linear theory where magnetic resonaces occur and some of them overlap.

The paper organized as follows. In Sect. 2, we derive the equations of motion in the linearized theory. In Sect. 2 we discuss the interaction of the GW and the magnetic field when the GW is parallel to the magnetic field. In Sect. 4 we discuss the same problem assuming that the $\mathrm{GW}$ is perpendicular to the magnetic field. The obtained results are discussed in Sect. 5

\section{Derivation of the equations of motion}

In the linearized approximation to general relativity the metric tensor is decomposed in the fashion

$g_{i j}=\eta_{i j}+h_{i j}$ 
where the elements $h_{i j}$ are small compared to unity. By imposing the condition

$\left(h_{i}^{j}-\delta_{i}^{j} h_{l}^{l}\right)_{; j}=0$

we reduce the vacuum field equations to homogeneous wave equations for all components of $h_{i}^{j}$. The gravitational field is then described by a symmetric, traceless, divergenceless tensor with two independent space components. Thus, the square of the line element is

$\mathrm{d} s^{2}=\left(\eta_{i j}+h_{i j}\right) \mathrm{d} x^{i} \mathrm{~d} x^{j}=\left(\mathrm{d} x^{0}\right)^{2}-\left(\mathrm{d} x^{a}\right)^{2}+h_{\alpha \beta} \mathrm{d} x^{\alpha} \mathrm{d} x^{\beta}$

where Greek indices take values 1, 2, 3 and Latin 0, 1, 2, 3 .

The components of the covariant four-velocity, consistent with the linearized theory, are

$u^{0} \equiv \frac{\mathrm{d} x^{0}}{\mathrm{~d} s} \cong u_{(M)}^{0}\left[1-\frac{1}{2} h_{\alpha \beta} u_{(M)}^{\alpha} u_{(M)}^{\beta}\right]$

$u^{\alpha} \equiv \frac{\mathrm{d} x^{\alpha}}{\mathrm{d} x^{0}}=\frac{\mathrm{d} x^{\alpha}}{\mathrm{d} x^{0}} \frac{\mathrm{d} x^{0}}{\mathrm{~d} s} \cong u_{(M)}^{0} \frac{v^{\alpha}}{c}\left[1-\frac{1}{2} h_{\alpha \beta} u_{(M)}^{\alpha} u_{(M)}^{\beta}\right]$

where $u^{0}, u^{\alpha}$ are the components of the four-velocity and the same quantities with the subscript $M$ distinguish the specialrelativistic Minkowski values.

The equations of motion of a test particle moving in the presence of an electromagnetic field in the space-time defined by Eq. (1) are given by

$\frac{\mathrm{d} u^{i}}{\mathrm{~d} s}+\Gamma_{j k}^{i} u^{j} u^{k}=\frac{e}{m c^{2}} F^{i k} u_{k}$

where the right-hand side is the inhomogeneous driving term determined by the electromagnetic field in the space-time defined by the Eq. (1).

For the metric (1), the non-zero Christoffel symbols are

$$
\begin{aligned}
& \Gamma_{\alpha \beta}^{0}=-\frac{1}{2} h_{\alpha \beta, 0}, \quad \Gamma_{0 \beta}^{\alpha}=\frac{1}{2} h_{\beta, 0}^{\alpha}, \\
& \Gamma_{\beta \gamma}^{\alpha}=\frac{1}{2}\left(h_{\beta, \gamma}^{\alpha}+h_{\gamma, \beta}^{\alpha}-h_{\beta \gamma}^{\alpha}\right) .
\end{aligned}
$$

From Eqs. (6), (4), (5) and (7), the equations of motions take the form:

$$
\begin{aligned}
& u_{(M)}^{0} u_{(M), 0}^{0}\left[1-h_{\alpha \beta} u_{(M)}^{\alpha} u_{(M)}^{\beta}\right]-\frac{1}{2}\left(u_{(M)}^{0}\right)^{2}\left[h_{\alpha \beta} u_{(M)}^{\alpha} u_{(M)}^{\beta}\right]_{, 0} \\
& -\frac{1}{2} h_{\alpha \beta, 0} u_{(M)}^{\alpha} u_{(M)}^{\beta}=\frac{e}{m c^{2}}\left(\eta^{0 l}-h^{0 l}\right. \\
& \left.-\frac{\eta^{0 l}}{2} h_{\alpha \beta} u_{(M)}^{\alpha} u_{(M)}^{\beta}\right)\left(F_{l 0} u^{0}+F_{l \alpha} u^{\alpha}\right)
\end{aligned}
$$

and

$$
\begin{aligned}
& u_{(M)}^{0} u_{(M), 0}^{\alpha}\left[1-h_{\alpha \beta} u_{(M)}^{\alpha} u_{(M)}^{\beta}\right]-\frac{1}{2} u_{(M)}^{0} u_{(M)}^{\alpha}\left[h_{\alpha \beta} u_{(M)}^{\alpha} u_{(M)}^{\beta}\right]_{, 0} \\
& +h_{\beta, 0}^{\alpha} u_{(M)}^{0} u_{(M)}^{\beta}+\frac{1}{2}\left(h_{\beta, \gamma}^{\alpha}+h_{\gamma, \beta}^{\alpha}-h_{\beta \gamma}^{\alpha}\right) u_{(M)}^{\beta} u_{(M)}^{\alpha}=\frac{e}{m c^{2}} \\
& \times\left(\eta^{\alpha l}-\frac{\eta^{\alpha l}}{2} h_{\alpha \beta} u_{(M)}^{\alpha} u_{(M)}^{\beta}-h^{\alpha l}\right)\left(F_{l 0} u_{(M)}^{0}+F_{l \alpha} u_{(M)}^{\alpha}\right)
\end{aligned}
$$$$
\text { (9) } \frac{\partial v^{2}}{\partial t}+\Omega v^{1}=h i \omega_{\mathrm{g}} v^{2}\left[1-\frac{v^{3}}{c}\right]
$$
terized by the wave vector isfy the conditions

Conditions (14) imply analyzed in the following two sections.

$F_{i j}=\left(\begin{array}{cccc}0 & 0 & 0 & 0 \\ 0 & 0 & -H_{3} & 0 \\ 0 & H_{3} & 0 & 0 \\ 0 & 0 & 0 & 0\end{array}\right)$

where $H^{3}=H=$ constant. aid of Eq. (16) yields:

$$
\frac{\partial v^{1}}{\partial t}-\Omega v^{2}=-h i \omega_{\mathrm{g}} v^{1}\left[1-\frac{v^{3}}{c}\right]
$$

Finally, the equations of motion (8) and (9), in the Newtonian and linearized limit, reduce to the equations:

$$
\begin{gathered}
\frac{\partial v^{i}}{\partial t}+\eta^{i k} h_{j k, t} v^{j}+\frac{1}{2} \eta^{i l}\left[h_{j l, k}+h_{k l, j}-h_{j k, l}\right] \\
\times v^{j} v^{k}=\frac{q}{m c}\left(\eta^{i a}-h^{i a}\right) F_{a l} v^{l} .
\end{gathered}
$$

To make further progress with the derived equations of motion (11), we consider the gravitational wave which is charac-

$k^{\alpha}=\frac{\omega}{c}(\sin (\theta), 0, \cos (\theta))$, and, $\frac{\omega^{2}}{c^{2}}=k^{a} k_{a}$

and one of two possible states of polarization given by

$$
\begin{aligned}
h_{i j}= & h_{0}\left(e_{i}^{1} e_{j}^{1}-e_{i}^{2} e_{j}^{1}\right) \\
& \times \exp \left[i \frac{\omega_{\mathrm{g}}}{c}\left(x^{1} \sin (\theta)+x^{3} \cos (\theta)-\text { const. }\right)\right]
\end{aligned}
$$

where $h_{0}$ is the amplitude of the gravitational wave and $\omega_{\mathrm{g}}=$ $2 \pi v_{\mathrm{g}}$ is the angular frequency of the GW.

The vectors $\boldsymbol{e}^{1}$ and $\boldsymbol{e}^{2}$ have space components only and sat-

$e^{1 \mu} e_{\mu}^{1}=e^{2 \mu} e_{\mu}^{2}=1$, with, $k^{\mu} e_{\mu}^{1}=k^{\mu} e_{\mu}^{2}=0$.

$\boldsymbol{e}_{\mu}^{1}=(\cos (\theta), 0,-\sin (\theta)), \boldsymbol{e}_{\mu}^{2}=(0,1,0)$.

Under the above consideration, we proceed to the following two cases: a) the GW is parallel to the magnetic field and b) the GW is perpendicular to the magnetic field, which will be

\section{The GW is parallel to the magnetic field}

We choose the electromagnetic field to be

We choose the gravitational wave to propagate parallel to the magnetic field e.g., in Eq. (13) we obtain $\theta=0$ and thus $h=h_{11}=-h_{22}=h_{0} \exp \left(\frac{i \omega_{\mathrm{g}}}{c}(z-c t)\right)$. Equation (11) with the 
$\frac{\partial v^{3}}{\partial t}=-i h \frac{1}{2 c} \omega_{\mathrm{g}}\left[\left(v^{1}\right)^{2}-\left(v^{2}\right)^{2}\right]$

where $\Omega=\frac{e H}{m c}$. To solve the system of Eqs. (17)-(19), we decompose the components of the 3 -velocity as follows:

$v^{1} \simeq v_{0}^{1}+v_{1}^{1}, \quad v^{2} \simeq v_{0}^{2}+v_{1}^{2}, \quad v^{3} \simeq 0+v_{1}^{3}$

where the subscript zero means zero order in the sense that $h_{0}=0$, while the subscript one means first order in the sense that $h_{0} \neq 0$.

The perturbed equations of motion are derived from Eqs. (17)-(20). Thus, after some straightforward calculations (see Appendix B), we find the solution (Macedo \& Nelson 1990):

$$
\begin{aligned}
v^{1} \simeq & v_{0 \mathrm{~T}} \cos (\Omega t+a)+h_{0} v_{0 \mathrm{~T}} \frac{\Omega-\omega_{\mathrm{g}}}{\left(2 \Omega-\omega_{\mathrm{g}}\right)} \\
& \times\left\{\cos \left[k_{\mathrm{g}} z+\left(\Omega-\omega_{\mathrm{g}}\right) t\right]-\cos \left(k_{\mathrm{g}} z-\Omega t\right)\right\}
\end{aligned}
$$

$$
\begin{aligned}
v^{2} \simeq & -v_{0 \mathrm{~T}} \sin (\Omega t+a)+h_{0} v_{0 \mathrm{~T}} \frac{\Omega-\omega_{\mathrm{g}}}{\left(2 \Omega-\omega_{\mathrm{g}}\right)} \\
& \times\left\{\sin \left[k_{\mathrm{g}} z+\left(\Omega-\omega_{\mathrm{g}}\right) t\right]-\sin \left(k_{\mathrm{g}} z-\Omega t\right)\right\}
\end{aligned}
$$

$$
\begin{aligned}
v^{3} \simeq & \frac{h_{0}}{2}\left(\frac{v_{0 \mathrm{~T}}^{2}}{c}\right) \exp \left(i k_{\mathrm{g}} z\right)\left\{\frac{\omega_{\mathrm{g}}^{2}}{4 \Omega^{2}-\omega_{\mathrm{g}}^{2}}-\left[\frac{\omega_{\mathrm{g}}}{\left(2 \Omega-\omega_{\mathrm{g}}\right)}\right.\right. \\
& \times \exp \left(i\left(2 \Omega-\omega_{\mathrm{g}}\right) t-\frac{\omega_{\mathrm{g}}}{\left(2 \Omega+\omega_{\mathrm{g}}\right)} \exp \left(i\left(2 \Omega+\omega_{\mathrm{g}}\right) t\right]\right\}
\end{aligned}
$$

where $v_{0 \mathrm{~T}}^{2}=v_{x}^{2}+v_{y}^{2}=$ constant and $a=$ constant.

It is evident that in Eqs. (21)-(23) if $h_{0}=0$, we obtain the components of the space velocity of the initial gyrating charged particle. If $h_{0} \neq 0$, the Eqs. (21)-(23) reveal that the gyrating charged particle diverts from its initial plane orbit and moves into a helical trajectory. Now the vector $\boldsymbol{v}=\left(v^{1}, v^{2}, v^{3}\right)$ does not move in a circle, but on the surface of a cone with its axis along the $\boldsymbol{H}$. From Eqs. (21)-(23) we conclude that the particle is accelerated at the resonace $\omega_{\mathrm{g}}=2 \Omega$. The existence of the resonace at $\omega_{\mathrm{g}}=2 \Omega$ in Eqs. (21)-(23) is due to the GW. Because of the resonance, the gyrating particle gains kinetic energy. Thus, if $E_{0}$ and $E_{1}$ are the kinetic energy of the particle before and after the interaction with the GW, respectively, the energy gained by the particle in a period, let's say $T$, is given by the average of the ration $\frac{E_{1}}{E_{0}}$ e.g.

$I=\frac{1}{T} \int_{0}^{T} \frac{E_{1}}{E_{0}} \mathrm{~d} t \approx 1-2 h_{0} \frac{\Omega-\omega_{\mathrm{g}}}{2 \Omega-\omega_{\mathrm{g}}} \cos \left(k_{\mathrm{g}} z\right)$.

Obviously, as $\omega_{\mathrm{g}}$ approaches $2 \Omega$, taking values between $\Omega$ and $2 \Omega$, the factor $\frac{\Omega-\omega_{\mathrm{g}}}{2 \Omega-\omega_{\mathrm{g}}}$, becomes negative, making the term multiplied by $h_{0}$ approach plus infinity with positive values. This suggests extra emission of cyclotron radiation which will change the spectra distribution of the radiation of the initial gyrating charged particle and transfer energy from the GW to the particle.

Integrating the Eqs. (21)-(23) we obtain the parametric equations of motion of the charged gyrating particle interacting with a GW in the presence of a constant magnetic field across the $z$-axis. These are

$$
\begin{aligned}
x_{(1)}(t)= & x_{(01)}+h_{0} v_{0 \mathrm{~T}} \frac{\Omega-\omega_{\mathrm{g}}}{2 \Omega-\omega_{\mathrm{g}}} \\
& \times\left\{\frac{\sin \left[k_{\mathrm{g}} z+\left(\Omega-\omega_{\mathrm{g}}\right) t\right]}{\Omega-\omega_{\mathrm{g}}}-\frac{\sin \left(k_{\mathrm{g}} z-\Omega t\right)}{\Omega}\right\} \\
y_{(1)}(t)= & \left.y_{(01)}-h_{0} v_{0 \mathrm{~T}} \frac{\Omega-\omega_{\mathrm{g}}}{2 \Omega-\omega_{\mathrm{g}}}\right) \\
& \times\left\{\frac{\cos \left[k_{\mathrm{g}} z+\left(\Omega-\omega_{\mathrm{g}}\right) t\right]}{\Omega-\omega_{\mathrm{g}}}+\frac{\cos \left(k_{\mathrm{g}} z-\Omega t\right)}{\Omega}\right\}
\end{aligned}
$$

and

$$
\begin{aligned}
z_{(1)}(t)= & z_{(01)}+\frac{h_{0}}{2} v_{0 \mathrm{~T}}\left(\frac{v_{0 \mathrm{~T}}}{c}\right) \exp \left(i k_{\mathrm{g} z}\right)\left\{\frac{t \omega_{\mathrm{g}}^{2}}{4 \Omega^{2}-\omega_{\mathrm{g}}^{2}}\right. \\
& +\frac{i}{2}\left[\frac { \omega _ { \mathrm { g } } } { ( 2 \Omega - \omega _ { \mathrm { g } } ) ^ { 2 } } \operatorname { e x p } \left(i\left(2 \Omega-\omega_{\mathrm{g}}\right) t\right.\right. \\
& \left.+\frac{\omega_{\mathrm{g}}}{\left(2 \Omega+\omega_{\mathrm{g}}\right)^{2}} \exp \left(i\left(2 \Omega+\omega_{\mathrm{g}}\right) t\right]\right\}
\end{aligned}
$$

where $x_{(01)}, y_{(01)}$ and $z_{(01)}$ are constants of integration.

The intensity of the radiation per solid angle per unit interval of frequency produced from a charge test particle moving in the presence of a magnetic field which interacts with the GW maybe obtained from the relation (Landau 1975):

$$
\begin{aligned}
\frac{\mathrm{d}^{2} I}{\mathrm{~d} \Omega_{a} \mathrm{~d} \omega^{\prime}}= & \frac{q^{2}\left(\omega^{\prime}\right)^{2}}{4 \pi^{2} c} \mid \int_{-\infty}^{\infty} \mathrm{d} t \exp \left(i \omega^{\prime}\left(t-\frac{\boldsymbol{n} \cdot \boldsymbol{R}}{c}\right)\right) \\
& \times\left.[\boldsymbol{n} \times(\boldsymbol{n} \times \boldsymbol{b})]\right|^{2}
\end{aligned}
$$

where $\omega^{\prime}$ is the frequency of the outgoing radiation, $\boldsymbol{n}=$ $\sin (\theta) \boldsymbol{i}+\cos (\theta) \boldsymbol{k}, \boldsymbol{R}$ is a vector that joins the charged particle with the observer, $\boldsymbol{b}$ is the velocity of the charge particle.

We carry out the integral of Eq. (28) neglecting terms of the order $\frac{v^{2}}{c^{2}}$. Thus, we find:

$$
\begin{aligned}
\frac{\mathrm{d}^{2} I}{\mathrm{~d} \Omega_{a} \mathrm{~d} \omega^{\prime}}= & \frac{q^{2} \varpi^{2}}{c} \sum_{-\infty}^{\infty} \delta(l \Omega-\varpi) \\
& \times\left\{\left[\cot ^{2}(\theta) J_{l}^{2}(\Phi)+\frac{v_{0 \mathrm{~T}}^{2}}{c^{2}}\left(J_{l}^{\prime}\right)^{2}(\Phi)\right]\right. \\
& \left.-4 h_{0} \cot ^{2}(\theta) \cos \left(k_{\mathrm{g}} z\right) \frac{\Omega-\omega_{\mathrm{g}}}{2 \Omega-\omega_{\mathrm{g}}} J_{l}^{2}(\Phi)\right\}
\end{aligned}
$$

where $\varpi=l \Omega\left[1+h_{0} \frac{v_{0 \mathrm{~T}}^{2} \omega^{2}}{2 c^{2}\left(2 \Omega^{2}-\omega_{g}^{2}\right)} \sin \left(k_{\mathrm{g}} z\right)\right]=l \Omega\left[1+O\left(1 / c^{2}\right)\right]$, $\Phi=\frac{\omega^{\prime}}{\Omega} \frac{v_{0 \mathrm{~T}}}{c} \sin (\theta), J_{j}$ is the Bessel function of first kind and $J_{l}^{\prime}$ its first ordinary derivative in terms of its argument. For simplicity we call

$$
L=\left[\cot ^{2}(\theta) J_{l}^{2}(\Phi)+\frac{v_{0 \mathrm{~T}}^{2}}{c^{2}}\left(J_{l}^{\prime}\right)^{2}(\Phi)\right]
$$

and

$T=-4 h_{0} \cot ^{2}(\theta) \cos \left(k_{\mathrm{g}} z\right) \frac{\Omega-\omega_{\mathrm{g}}}{2 \Omega-\omega_{\mathrm{g}}} J_{l}^{2}(\Phi)$. 
It is evident that, as $\omega_{\mathrm{g}}$ approaches $2 \Omega^{+}$, the factor $\frac{\Omega-\omega_{\mathrm{g}}}{2 \Omega-\omega_{\mathrm{g}}} \rightarrow$ $\infty$ making the term $T$ tend to minus infinity. But, if $\omega_{\mathrm{g}}$ approaches $2 \Omega$ taking values between $\Omega$ and $2 \Omega$, the factor $\frac{\Omega-\omega_{g}}{2 \Omega-\omega_{g}}$ becomes negative, making the term $T$ approach plus infinity with positive values. Also, from Eq. (31), we see that the divergence of the term $T$ is faster as we approach the source producing GW. Nevertheless, in the linearized theory of gravitation, we have to approach the resonant in such a way that the term $T$ remains always below the term $L$, otherwise the linear theory breaks down.

\section{The GW is perpendicular to the magnetic field}

We shall now consider the case where the GW propagates perpendicular to the unperturbed magnetic field $H^{\mu}=\left(0,0, H^{3}\right)=$ const. Thus, in a reference frame where $H^{\mu}$ has the $z$-direction (as for the previous case), but the GW propagates along the $x$-direction, the two non-vanishing components of the GW are given by Eqs. (13) setting $\theta=\pi / 2$ and

$h_{33}=-h_{22}=h_{0} \exp \frac{i \omega}{c}(x-c t)$.

Subsequently, from Eqs. (11), (20) and (32) we obtain the following equations of motion:

$$
\begin{aligned}
v^{1}= & v_{0}^{1}+v_{1}^{1}=v_{0 \mathrm{~T}} \cos (\Omega t+a)+v_{0 \mathrm{~T}} h_{0}\{C \cos (\Omega t) \\
& \left.-\left[A \cos \left(k_{\mathrm{g}} z-\omega_{\mathrm{g}} t\right)-B \sin \left(k_{\mathrm{g}} z-\omega_{\mathrm{g}} t\right)\right]\right\}
\end{aligned}
$$$$
v^{2}=v_{0}^{2}+v_{1}^{2}=-v_{0 \mathrm{~T}} \sin (\Omega t+a)-v_{0 \mathrm{~T}} h_{0}\{C \sin (\Omega t)
$$$$
\left.+\left[A \sin \left(k_{\mathrm{g}} z-\omega_{\mathrm{g}} t\right)-B \cos \left(k_{\mathrm{g}} z-\omega_{\mathrm{g}} t\right)\right]\right\}
$$

and

$v^{3}=v_{0}^{3}+v_{1}^{3}=\sigma-\sigma\left(1-\frac{\sigma}{c}\right) h_{22}$

where, $\sigma=$ constant and may be chosen equal zero,

$$
\begin{aligned}
C= & \frac{\Omega^{2}}{\left(\Omega-\omega_{\mathrm{g}}\right)\left(2 \Omega-\omega_{\mathrm{g}}\right)}\left[1+\left(\frac{v_{0 \mathrm{~T}}}{c}\right) \frac{\omega_{\mathrm{g}}^{2}\left(2 \Omega-\omega_{\mathrm{g}}\right)}{\Omega\left(\Omega+\omega_{\mathrm{g}}\right)\left(3 \Omega-\omega_{\mathrm{g}}\right)}\right](36) \\
A= & \frac{\Omega}{\Omega-\omega_{\mathrm{g}}}-\frac{\Omega}{2 \Omega-\omega_{\mathrm{g}}} \cos (\Omega t+2 a) \\
& +\frac{v_{0 \mathrm{~T}}}{4 c}\left[\frac{\omega_{\mathrm{g}}}{\Omega-\omega_{\mathrm{g}}}-\frac{\omega_{\mathrm{g}}\left(3 \Omega+\omega_{\mathrm{g}}\right)}{\left(\Omega+\omega_{\mathrm{g}}\right)\left(3 \Omega-\omega_{\mathrm{g}}\right)} \cos (2 \Omega t+2 a)\right]
\end{aligned}
$$

and

$$
\begin{aligned}
B= & \frac{\Omega-\omega_{\mathrm{g}}}{2 \Omega-\omega_{\mathrm{g}}} \sin (\Omega t+2 a) \\
& -\frac{v_{0 \mathrm{~T}}}{2 c} \frac{\omega_{\mathrm{g}}^{2}}{\left(\Omega+\omega_{\mathrm{g}}\right)\left(3 \Omega-\omega_{\mathrm{g}}\right)} \sin (2 \Omega t+2 a) .
\end{aligned}
$$

Obviously, if in Eqs. (33)-(35) $h_{0}=0$, then we obtain the space velocities of the initial gyrating charged particle.
If $h_{0} \neq 0$, then we have gyrating motion again, but magnetic resonances appear at $\omega_{\mathrm{g}}= \pm \Omega, \omega_{\mathrm{g}}=2 \Omega$ and $\omega_{\mathrm{g}}=3 \Omega$.

The existence of the above resonances in Eqs. (33)-(35) is due to the GW. Because of those resonances, the gyrating particle gains kinetic energy. Thus, as in Sect. 3, we verify that the energy gained by the particle in a period, let say $T$, is given averaging the ratio $\frac{E_{1}}{E_{0}}$ e.g.

$I_{2}=\frac{1}{T} \int_{0}^{T} \frac{E_{1}}{E_{0}} \mathrm{~d} t \approx 1+2 h_{0} C \approx 1+2 h_{0} \frac{\Omega^{2}}{\left(\Omega-\omega_{\mathrm{g}}\right)\left(2 \Omega-\omega_{\mathrm{g}}\right)}$.

Obviously, if $\omega_{\mathrm{g}}$ approaches $2 \Omega$ from the right or $\Omega$ from the left, the factor $\frac{\Omega^{2}}{\left(\Omega-\omega_{\mathrm{g}}\right)\left(2 \Omega-\omega_{\mathrm{g}}\right)}$ becomes positive, making the term multiplied by $h_{0}$ approach plus infinity with positive values, suggesting changes to the spectra distribution of the radiation of the initial gyrating charged particle.

Integrating Eqs. (33)-(35) we derive the parametric equations of motion which are:

$x(t)=\frac{v_{0 \mathrm{~T}}}{c} \sin (\Omega t)+h_{0} v_{0 \mathrm{~T}}\left[\frac{\sin (\Omega t)}{\Omega} C+X_{h}\right]$

$y(t)=\frac{v_{0 \mathrm{~T}}}{c} \cos (\Omega t)+h_{0} v_{0 \mathrm{~T}}\left[\frac{\cos (\Omega t)}{\Omega} C+Y_{h}\right]$

$z(t)=\sigma t+i \frac{\sigma}{\omega_{\mathrm{g}}}\left(1-\frac{\sigma}{c}\right) h_{22}$

where $\sigma=$ constant and may be chosen equal to zero; the expression for $X_{h}$ and $Y_{h}$ are given explicitly in the Appendix A.

In the Eqs. (40) and (41) a drift term, with resonances at $\omega_{\mathrm{g}}=\Omega, \omega_{\mathrm{g}}=2 \Omega$, is present. This drift term can generate electric currents. Those currents are sources of secondary electromagnetic waves. For further details see Macedo \& Nelson (1990).

Following the same procedure as in Sect. 3, Eq. (28) reduce to

$$
\begin{aligned}
\frac{\mathrm{d}^{2} I}{\mathrm{~d} \Omega_{a} \mathrm{~d} \omega^{\prime}}= & \frac{q^{2} \varpi^{2}}{c} \sum_{-\infty}^{\infty} \delta(l \Omega-\varpi) \\
& \times\left\{\left[\cot ^{2}(\theta) J_{l}^{2}(\Phi)+\frac{v_{0 \mathrm{~T}}^{2}}{c^{2}}\left(J_{l}^{\prime}\right)^{2}(\Phi)\right]\right. \\
& -h_{0} \cos (2 \theta) \cot ^{2}(\theta)\left(\frac{v_{0 \mathrm{~T}}}{c}\right) \\
& \left.\times \frac{2 \Omega^{2}}{\left(\Omega-\omega_{\mathrm{g}}\right)\left(2 \Omega-\omega_{\mathrm{g}}\right)} J_{l}^{2}(\Phi)\right\} .
\end{aligned}
$$

As in Sect. 3, we call

$L=\left[\cot ^{2}(\theta) J_{l}^{2}(\Phi)+\frac{v_{0 \mathrm{~T}}^{2}}{c^{2}}\left(J_{l}^{\prime}\right)^{2}(\Phi)\right]$

and

$T_{2}=-h_{0} \cos (2 \theta) \cot ^{2}(\theta)\left(\frac{v_{0 \mathrm{~T}}}{c}\right) \frac{2 \Omega^{2}}{\left(\Omega-\omega_{\mathrm{g}}\right)\left(2 \Omega-\omega_{\mathrm{g}}\right)} J_{l}^{2}(\Phi)$. 
Now the term $T_{2}$ has two magnetic resonances. It is evident that, as $\omega_{\mathrm{g}}$ approaches $2 \Omega^{-}$or $\Omega^{+}$and $\theta \in\left(0, \frac{\pi}{4}\right)$, the term $T_{2}$ tends to plus infinity. Also, from Eq. (45), we see that the divergence of the term $T_{2}$ becomes faster as we approach the source producing the GW as we are dealing with ultra relativistic particles where the ratio $\frac{v_{0 T}}{c}$ takes higher values. Again, as in in the Sect. 3, in the linearized theory of gravitation we have to approach to the resonances carefully, in the sense that the term $T_{2}$ should not exceed the term $L$, otherwise the theory breaks down.

\section{Conclusions}

In this article we pose the following problem. If $h_{0}=0$, we reproduce a well known formula for the spectrum distribution of a gyrating charged particle in both cases e.g. when the GW is parallel and perpendicular to the magnetic field. In this case the angular distribution of the gyro radiation is highly anisotropic. The radiation is concentrated mainly in the plane of the orbit.

If $h_{0} \neq 0$, we are dealing with the interaction of a GW with a gyrating charged particle. We have distinguished two cases:

(a) The GW propagates parallel to the constant magnetic field:

Because of the GW, the gyrating charged particle is diverted from its initial plane orbit and starts to move across a helical trajectory. Also, because of the GW, the spectrum of the produced cyclotron radiation is isolated by a factor proportional to $h_{0}$, namely $T$, in which a resonance at $\omega_{\mathrm{g}}=2 \Omega$ appears. If $\omega_{\mathrm{g}}$ approaches $2 \Omega$ taking values in the interval $I_{1}=(\Omega, 2 \Omega)$, the term $T$ approaches plus infinity, indicating that the existence of the resonant interaction between the charged particle and the GW can lead to a strong emission of cyclotron radiation, even in the linear theory of gravity. The suggested mechanism of cyclotron radiation could be useful to astrophysicists, especially they make simultaneous observations of the cyclotron radiation and the GW so obtained. Therefore, knowing that astrophysicists are looking to detect GWs at frequencies between $v_{\mathrm{g}}=\left(10^{2}-10^{3}\right) \mathrm{Hz}$ (Cutler Thorne 2002), magnetic resonance may occur whenever the magnetic fields are between $H \approx(1 .-2) G.\left(v_{\mathrm{g}}=10^{2} \mathrm{~Hz}\right), H \approx(10-21) G$ $\left(v_{\mathrm{g}}=10^{3} \mathrm{~Hz}\right)$ and in both cases the $T$ term becomes positive and comparable to the $L$ term. But, if we want to use electromagnetic radiation for indirect detection of $\mathrm{GW}$, then other frequencies are also important, e.g. lower frequencies of $v_{\mathrm{g}}=(1-10) \mathrm{Hz}$ coming from binary neutron stars before coalescence. The corresponding magnetic fields are $H \approx$ $(0.01-0.02) \mathrm{G}\left(v_{\mathrm{g}}=1 \mathrm{~Hz}\right), H \approx(0.1-0.2) \mathrm{G}\left(v_{\mathrm{g}}=10 \mathrm{~Hz}\right)$ or high frequencies $v_{\mathrm{g}}=\left(10^{3}-10^{4}\right) \mathrm{Hz}$, due to supernovae explosions, normal modes of pulsating neutron stars or star-size black holes. In the later case the corresponding magnetic fields are $H \approx(107-214) \mathrm{G}$.

Outside the interval $I_{1}$ the particle seems to lose energy due to destructive interference of the two oscillators e.g. the sinusoidal gravitational wave and the gyrating particle. In this case, the actual loss described by the negative term $T$ is of the order of $h_{0}$.

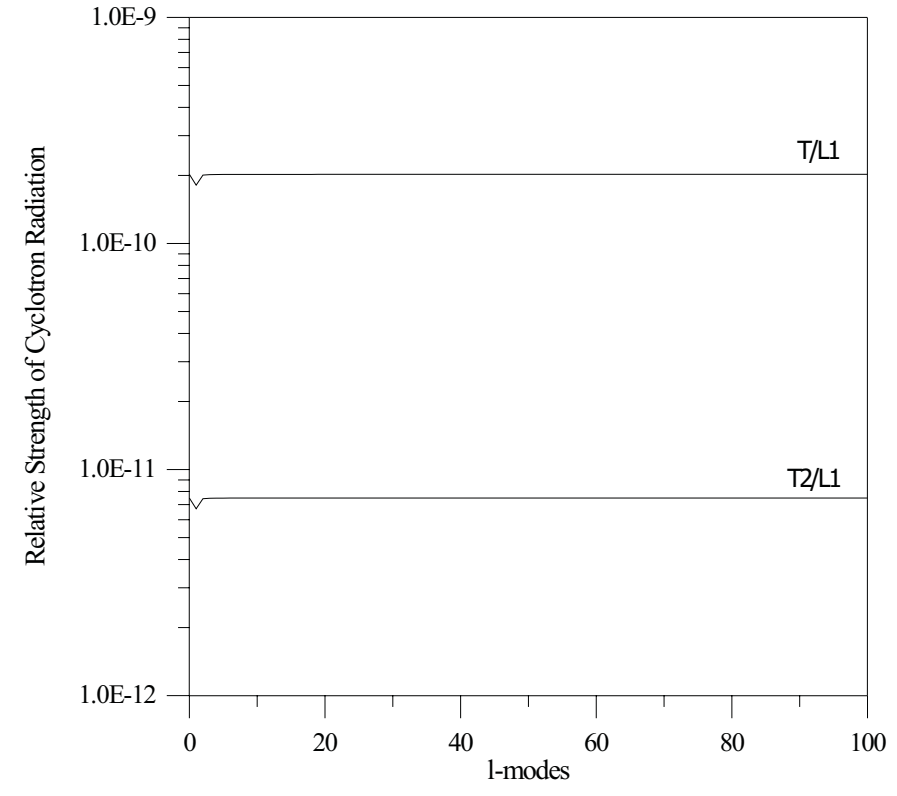

Fig. 1. The strengths of cyclotron radiation in the cases where $H$ is parallel to the $k(T / L 1)$ and $H$ is perpendicular to the $k$

(b) The GW propagates in the $x$-direction e.g. perpendicular to the constant magnetic field.

In this case, we verify that 1 ) the gyrating particle remains on the initial plane of orbit described by Eqs. (33)-(35) and for a certain $\theta=\theta_{0}$, let's say $\theta_{0} \in\left(0, \frac{\pi}{4}\right)$, at $\omega_{\mathrm{g}}=\Omega$ and $\omega_{\mathrm{g}}=2 \Omega$ the corresponding term $T_{2}$ diverges. 2) If $\omega_{\mathrm{g}}$ approaches $\Omega$ from the left or $2 \Omega$ from the right, whereas $\theta_{0} \in\left(\frac{\pi}{4}, \frac{\pi}{2}\right)$, the term $T_{2}$ becomes positive, and approaches plus infinity, unless $\theta_{0} \in\left(0, \frac{\pi}{4}\right)$. For the frequencies mentioned above, two magnetic resonaces may occur for the same values of $\omega_{\mathrm{g}}$ at the same range of the magnetic field. But if $\omega_{\mathrm{g}}$ approaches one or other resonance, taking values in interval $I_{1}$, where $\theta_{0} \in\left(\frac{\pi}{4}, \frac{\pi}{2}\right)$, the term $T_{2}$ becomes negative (unless $\theta_{0} \in\left(0, \frac{\pi}{4}\right)$ ), indicating that the particle loses energy due to the same reason mentioned in paragraph (a). Another interesting feature is that the term $T_{2}$ is proportional to the ratio $\frac{v_{0 \mathrm{~T}}}{c}$, which means that high relativist particles support the term $T_{2}$ to become more significant. Nevertheless, in both cases, the strength of the cyclotron radiation described by the ratios $\frac{T}{L_{1}}$ and $\frac{T_{2}}{L_{1}}$ remain constant for large values of 1 (high frequencies). This may seen in Fig. 1 where we plot the mentioned above ratios for $\theta_{0}=80^{\circ}$, obtaining the amplitude of the GW to be $h_{0}=10^{-21}$ and its frequency $v_{\mathrm{g}}=10^{3}$. However, we have to point out that, in both cases, the terms multiplied by $h_{0}$ should not exceed the term $L$, otherwise the linearized theory of gravity breaks down.

In the nonlinear theory, the problem could be more interesting. The problem somehow has been examined from a dynamical point of view, considering the equations of motion from a Hamiltonian, (Varvoglis \& Papadopoulos 1992; Kleidis et al. 1993; Kleidis et al. 1995 and Kleidis et al. 1996), and integrating them numerically. In this case, the interaction of the GW 
with a gyrating charged particle exhibits resonances and in several cases chaotic behavior. We intend to discuss the problem in non-linear theory in a forthcoming paper.

Acknowledgements. The author would like to thank Loukas Vlahos, Kostas Kokkotas L. Witten and Nik Stergioulas for their comments, criticism and beneficial discussions.

\section{Appendix A}

In the Eqs. (33)-(35) the $X_{h}$ and $Y_{h}$ are:

$$
\begin{aligned}
& X_{h}=-\left\{-\frac{\Omega}{\omega_{\mathrm{g}}\left(\Omega-\omega_{\mathrm{g}}\right)} \sin \left(k_{\mathrm{g}} x-\omega_{\mathrm{g}} t\right)-\frac{\Omega}{2\left(2 \Omega-\omega_{\mathrm{g}}\right)}\right. \\
& \times\left[-\frac{1}{\Omega+\omega_{\mathrm{g}}} \sin \left[k_{\mathrm{g}} x-\left(\Omega+\omega_{\mathrm{g}}\right) t\right]\right. \\
& \left.+\frac{1}{\Omega-\omega_{\mathrm{g}}} \sin \left[k_{\mathrm{g}} x+\left(\Omega-\omega_{\mathrm{g}}\right) t\right]\right] \\
& +\frac{\Omega-\omega_{\mathrm{g}}}{2\left(2 \Omega-\omega_{\mathrm{g}}\right)}\left[\frac{1}{\Omega+\omega_{\mathrm{g}}} \sin \left[k_{\mathrm{g}} x-\left(\Omega+\omega_{\mathrm{g}}\right) t\right]\right. \\
& \left.+\frac{1}{\Omega-\omega_{\mathrm{g}}} \sin \left[k_{\mathrm{g}} x+\left(\Omega-\omega_{\mathrm{g}}\right) t\right]\right] \\
& +\frac{v_{0 \mathrm{~T}}}{4 c}\left[-\frac{1}{\left(\Omega-\omega_{\mathrm{g}}\right)} \sin \left(k_{\mathrm{g}} x-\omega_{\mathrm{g}} t\right)\right. \\
& -\frac{\omega_{\mathrm{g}}\left(3 \Omega+\omega_{\mathrm{g}}\right)}{2\left(\Omega+\omega_{\mathrm{g}}\right)\left(3 \Omega-\omega_{\mathrm{g}}\right)}\left[-\frac{1}{2 \Omega+\omega_{\mathrm{g}}}\right. \\
& \times \sin \left[k_{\mathrm{g}} x-\left(2 \Omega+\omega_{\mathrm{g}}\right) t\right]+\frac{1}{2 \Omega-\omega_{\mathrm{g}}} \\
& \left.\times \sin \left[k_{\mathrm{g}} x+\left(2 \Omega-\omega_{\mathrm{g}}\right) t\right]\right] \\
& +\frac{\omega_{\mathrm{g}}^{2}}{2\left(\Omega+\omega_{\mathrm{g}}\right)\left(3 \Omega+\omega_{\mathrm{g}}\right)}\left[\frac{1}{2 \Omega+\omega_{\mathrm{g}}}\right. \\
& \times \sin \left[k_{\mathrm{g}} x-\left(2 \Omega+\omega_{\mathrm{g}}\right) t\right]+\frac{1}{2 \Omega-\omega_{\mathrm{g}}} \\
& \left.\times \sin \left[k_{\mathrm{g}} x+\left(2 \Omega-\omega_{\mathrm{g}}\right) t\right]\right] \\
& -\sin k_{\mathrm{g}} x\left\{\frac{\Omega}{\omega_{\mathrm{g}}\left(\Omega-\omega_{\mathrm{g}}\right)}-\frac{\Omega\left(\Omega-2 \omega_{\mathrm{g}}\right)}{\left(2 \Omega-\omega_{\mathrm{g}}\right)\left(\Omega^{2}-\omega_{\mathrm{g}}^{2}\right)}\right. \\
& +\frac{2 \Omega \omega_{\mathrm{g}}^{2}}{\left(\Omega+\omega_{\mathrm{g}}\right)\left(3 \Omega-\omega_{\mathrm{g}}\right)\left(4 \Omega^{2}-\omega_{\mathrm{g}}^{2}\right)} \\
& +\frac{v_{0 \mathrm{~T}}}{4 c}\left[\frac{1}{\Omega-\omega_{\mathrm{g}}}+\frac{\omega_{\mathrm{g}}^{2}\left(3 \Omega+\omega_{\mathrm{g}}\right)}{\left(2 \Omega+\omega_{\mathrm{g}}\right)\left(3 \Omega-\omega_{\mathrm{g}}\right)\left(4 \Omega^{2}-\omega_{\mathrm{g}}^{2}\right)}\right. \\
& \left.\left.+\frac{2 \Omega \omega_{\mathrm{g}}^{2}}{\left(\Omega+\omega_{\mathrm{g}}\right)\left(3 \Omega-\omega_{\mathrm{g}}\right)\left(4 \Omega^{2}-\omega_{\mathrm{g}}^{2}\right)}\right]\right\}
\end{aligned}
$$

and

$$
\begin{aligned}
& Y_{h}=-\left\{-\frac{C}{\Omega}+\frac{\Omega}{\omega_{\mathrm{g}}\left(\Omega-\omega_{\mathrm{g}}\right)} \cos \left(k_{\mathrm{g}} x-\omega_{\mathrm{g}} t\right)-\frac{\Omega}{2\left(2 \Omega-\omega_{\mathrm{g}}\right)}\right. \\
& \times\left[\frac{\cos \left(k_{\mathrm{g}} x-\left(\Omega-\omega_{\mathrm{g}}\right) t\right)}{\Omega-\omega_{\mathrm{g}}}+\frac{\cos \left(k_{\mathrm{g}} x-\left(\Omega+\omega_{\mathrm{g}}\right) t\right)}{\Omega+\omega_{\mathrm{g}}}\right. \\
& +\frac{\Omega-\omega_{\mathrm{g}}}{2\left(2 \Omega-\omega_{\mathrm{g}}\right)}\left[\frac{\cos \left(k_{\mathrm{g}} x-\left(\Omega-\omega_{\mathrm{g}}\right) t\right)}{\Omega-\omega_{\mathrm{g}}}\right. \\
& \left.-\frac{\cos \left(k_{\mathrm{g}} x-\left(\Omega+\omega_{\mathrm{g}}\right) t\right)}{\Omega+\omega_{\mathrm{g}}}\right]-\cos \left(k_{\mathrm{g}} x\right) \\
& \times\left[\frac{\Omega}{\omega_{\mathrm{g}}\left(\Omega-\omega_{\mathrm{g}}\right)}-\frac{\Omega^{2}}{\left(2 \Omega-\omega_{\mathrm{g}}\right)\left(\Omega^{2}-\omega_{\mathrm{g}}^{2}\right)}\right. \\
& \left.+\frac{\omega_{\mathrm{g}}}{\left(2 \Omega-\omega_{\mathrm{g}}\right)\left(\Omega+\omega_{\mathrm{g}}\right)}\right] \\
& +\frac{v_{0 \mathrm{~T}}}{4 c}\left[\frac{1}{\left(\Omega-\omega_{\mathrm{g}}\right)} \cos \left(k_{\mathrm{g}} x-\omega_{\mathrm{g}} t\right)+\frac{2 \omega_{\mathrm{g}}}{\left(\Omega-\omega_{\mathrm{g}}\right)\left(3 \Omega-\omega_{\mathrm{g}}\right)}\right. \\
& \times \sin \left(k_{\mathrm{g}} x-\omega_{\mathrm{g}} t\right) \sin (2 \Omega t)-\frac{\omega_{\mathrm{g}}\left(3 \Omega+\omega_{\mathrm{g}}\right)}{2\left(\Omega+\omega_{\mathrm{g}}\right)\left(3 \Omega-\omega_{\mathrm{g}}\right)} \\
& \times\left[\frac{\cos \left(k_{\mathrm{g}} x-\left(2 \Omega-\omega_{\mathrm{g}}\right) t\right)}{2 \Omega-\omega_{\mathrm{g}}}+\frac{\cos \left(k_{\mathrm{g}} x-\left(2 \Omega+\omega_{\mathrm{g}}\right) t\right)}{2 \Omega+\omega_{\mathrm{g}}}\right] \\
& \left.-\cos \left(k_{\mathrm{g}} x\right)\left[\frac{1}{\Omega-\omega_{\mathrm{g}}}-\frac{2 \Omega \omega_{\mathrm{g}}\left(3 \Omega+\omega_{\mathrm{g}}\right)}{\left(\Omega+\omega_{\mathrm{g}}\right)\left(3 \Omega-\omega_{\mathrm{g}}\right)\left(4 \Omega^{2}-\omega_{\mathrm{g}}^{2}\right)}\right]\right\} \text {. }
\end{aligned}
$$

\section{Appendix B}

We start with Eqs. (17)-(19)

$$
\begin{aligned}
& \frac{\partial v^{1}}{\partial t}-\Omega v^{2}=-h i \omega_{\mathrm{g}} v^{1}\left[1-\frac{v^{3}}{c}\right] \\
& \frac{\partial v^{2}}{\partial t}+\Omega v^{1}=h i \omega_{\mathrm{g}} v^{2}\left[1-\frac{v^{3}}{c}\right] \\
& \frac{\partial v^{3}}{\partial t}=-i h \frac{1}{2 c} \omega_{\mathrm{g}}\left[\left(v^{1}\right)^{2}-\left(v^{2}\right)^{2}\right] .
\end{aligned}
$$

We write Eqs. (48), (49) as follows:

$\frac{\partial\left(v^{1}+i v^{2}\right)}{\partial t}+\Omega\left[v^{1}+i v^{2}\right]=h i \omega_{\mathrm{g}}\left(v^{1}-i v^{2}\right)\left[1-\frac{v^{3}}{c}\right]$.

In order to solve the Eq. (51), we decompose the components of the 3-velocity as follows:

$v^{1} \simeq v_{0}^{1}+v_{1}^{1}, \quad v^{2} \simeq v_{0}^{2}+v_{1}^{2}, \quad v^{3} \simeq 0+v_{1}^{3}$

where the subscript zero means zero order in the sense that $h_{0}=0$, while the subscript one means first order in the sense that $h_{0} \neq 0$.

We substitute Eqs. (52) into Eq. (51) and the perturbed equation now reads:

$$
\begin{array}{r}
\frac{\partial\left[\left(v_{0}^{1}+v_{1}^{1}\right)+i\left(v_{0}^{2}+i v_{1}^{2}\right)\right]}{\partial t}+i \Omega\left[\left(v_{0}^{1}+v_{1}^{1}\right)+i\left(v_{0}^{2}+v_{1}^{2}\right)\right] \\
=h i \omega_{\mathrm{g}}\left[\left(v_{0}^{1}+v_{1}^{1}\right)-i\left(v_{0}^{2}+v_{1}^{2}\right]\left[1-\frac{v_{1}^{3}}{c}\right] .\right.
\end{array}
$$




\section{Zero order equations}

$\frac{\partial\left(v_{0}^{1}+i v_{0}^{2}\right)}{\partial t}=-i \Omega\left(v_{0}^{1}+i v_{0}^{2}\right)$

This yields

$v_{0}^{1}=v_{0 \mathrm{~T}} \cos (\Omega t+a)$ and $v_{0}^{2}=-v_{0 \mathrm{~T}} \sin (\Omega t+a)$

where $a=$ constant and $v_{0 \mathrm{~T}}^{2}=v_{x}^{2}+v_{y}^{2}=$ constant.

\section{First order equations}

From Eq. (53), we have

$$
\frac{\partial\left(v_{1}^{1}+i v_{1}^{2}\right)}{\partial t}+i \Omega\left(v_{1}^{1}+i v_{1}^{2}\right)=h i \omega_{\mathrm{g}}\left(v_{0}^{1}-i v_{0}^{2}\right)
$$

Notice that on the right hand side, the factor $h\left(v_{0}^{1}-i v_{0}^{2}\right)$ gives

$$
\begin{aligned}
h\left(v_{0}^{1}-i v_{0}^{2}\right) & =h_{0} v_{0 \mathrm{~T}} \mathrm{e}^{i\left(k_{\mathrm{g}} z-\omega_{\mathrm{g}} t\right)}[\cos (\Omega t+a)-i \sin (\Omega t+a)] \\
& =h_{0} v_{0 \mathrm{~T}} \mathrm{e}^{i\left(k_{\mathrm{g}} z-\omega_{\mathrm{g}} t\right)} \mathrm{e}^{i(\Omega t+a)} .
\end{aligned}
$$

Now from Eqs. (56) and (57) we have

$\frac{\partial\left(v_{1}^{1}+i v_{1}^{2}\right)}{\partial t}+i \Omega\left(v_{1}^{1}+i v_{1}^{2}\right)=h_{0} v_{0 \mathrm{~T}} \mathrm{e}^{i\left(k_{\mathrm{g}} z+a\right)} \mathrm{e}^{i t\left(\Omega-\omega_{\mathrm{g}}\right)}$.

We treat Eq. (58) as an ordinary first order differential equation with the initial conditions: if $t=0$ then $\left(v_{1}^{1}+i v_{1}^{2}\right)=0$. Thus, we have

$$
\begin{aligned}
\left(v_{1}^{1}+i v_{1}^{2}\right) & =\mathrm{e}^{-i t \Omega}\left\{C+i h_{0} v_{0 \mathrm{~T}} \omega_{\mathrm{g}} \mathrm{e}^{i\left(k_{\mathrm{g}} z+a\right)} \int \mathrm{e}^{i t\left(\Omega-\omega_{\mathrm{g}}\right)} \mathrm{e}^{i \int \Omega \mathrm{d} t} \mathrm{~d} t\right\} \\
& =\mathrm{e}^{-i t \Omega}\left\{C+i h_{0} v_{0 \mathrm{~T}} \omega_{\mathrm{g}} \mathrm{e}^{i\left(k_{\mathrm{g}} z+a\right)} \int \mathrm{e}^{i t\left(2 \Omega-\omega_{\mathrm{g}}\right)} \mathrm{d} t\right\} \\
& =\mathrm{e}^{-i t \Omega}\left\{C+i h_{0} v_{0 \mathrm{~T}} \frac{\omega_{\mathrm{g}}}{2 \Omega-\omega_{\mathrm{g}}} \mathrm{e}^{i\left(k_{\mathrm{g}} z+a\right)} \mathrm{e}^{i t\left(2 \Omega-\omega_{\mathrm{g}}\right)}\right\} \cdot(59)
\end{aligned}
$$

Upon consideration of the initial conditions we have

$$
C=-h_{0} v_{0 \mathrm{~T}} \frac{\omega_{\mathrm{g}}}{2 \Omega-\omega_{\mathrm{g}}} \mathrm{e}^{i\left(k_{\mathrm{g}} z+a\right)} .
$$

Eventually, from the Eqs. (59) and (60) we obtain

$$
\left(v_{1}^{1}+i v_{1}^{2}\right)=h_{0} v_{0 \mathrm{~T}} \frac{\omega_{\mathrm{g}}}{2 \Omega-\omega_{\mathrm{g}}} \mathrm{e}^{i\left(k_{\mathrm{g}} z+a\right)}\left[\mathrm{e}^{i t\left(\Omega-\omega_{\mathrm{g}}\right)}-\mathrm{e}^{-i t \Omega}\right]
$$

or

$$
v_{1}^{1}=h_{0} v_{0 \mathrm{~T}} \frac{\omega_{\mathrm{g}}}{2 \Omega-\omega_{\mathrm{g}}}\left\{\cos \left[k_{\mathrm{g}} z+\left(\Omega-\omega_{\mathrm{g}}\right) t\right]-\cos \left(k_{\mathrm{g}} z-\Omega t\right)\right\}
$$

$$
v_{1}^{2}=h_{0} v_{0 \mathrm{~T}} \frac{\omega_{\mathrm{g}}}{2 \Omega-\omega_{\mathrm{g}}}\left\{\sin \left[k_{\mathrm{g}} z+\left(\Omega-\omega_{\mathrm{g}}\right) t\right]-\sin \left(k_{\mathrm{g}} z-\Omega t\right)\right\}
$$

Furthermore, following the same method, from Eq. (19) we find

$$
\begin{aligned}
v_{1}^{3}= & \frac{h_{0}}{2} v_{0 \mathrm{~T}}\left(\frac{v_{0 \mathrm{~T}}}{c}\right) \exp \left(i k_{\mathrm{g}} z\right)\left\{\frac{\omega_{\mathrm{g}}^{2}}{4 \Omega^{2}-\omega_{\mathrm{g}}^{2}}-\left[\frac{\omega_{\mathrm{g}}}{\left(2 \Omega-\omega_{\mathrm{g}}\right)}\right.\right. \\
& \times \exp \left(i\left(2 \Omega-\omega_{\mathrm{g}}\right) t-\frac{\omega_{\mathrm{g}}}{\left(2 \Omega+\omega_{\mathrm{g}}\right)} \exp \left(i\left(2 \Omega+\omega_{\mathrm{g}}\right) t\right]\right\}
\end{aligned}
$$

\section{References}

Brodin, G., \& Marklund M. 1999, Phys. Rev. Lett., 82, 3012

Cutler, C., \& Thorne, K. [gr-qc/0204090]

Kleidis, K., Varvoglis, H., \& Papadopoulos, D. 1993, A\&A, 275, 309

Kleidis, K., Varvoglis, H., Papadopoulos, D., \& Esposito F. P. 1995, A\&A, 294, 313

Kleidis, K., Varvoglis, H., \& Papadopoulos D. 1996, Class. Quantum. Grav., 13, 2547

Landau, L. D., \& Lifshitz, E. M. 1975, The Classical Theory of Fields (Pergamon Press)

Macedo, P. G., \& Nelson, A. G. 1982, Phys. Rev. D., 28, 2382

Macedo, P. G., \& Nelson, A. G. 1990, ApJ, 362, 584

Marklund, M., Brodin, G., \& Dunsby, P. K. S. 2000, ApJ, 536, 875

Papadopoulos, D., \& Esposito, F. P. 1981, ApJ, 248, 783

Papadopoulos, D., \& Esposito, F. P. 1982, ApJ, 257, 10

Servin, M., Brodin, G., \& Marklund, M. 2001, Phys. Rev. D., 64, 024013

Tsagas, C. G. 2001, Phys. Rev. Lett., 86, 5421

van Holten J.-W. [gr-qc/9906117]

Varvoglis, H., \& Papadopoulos, D. 1992, A\&A, 261, 664 